\title{
Lipomatous Metaplasia after Severe and Chronic Cutaneous Inflammation
}

\author{
Kilian Eyerich $^{\mathrm{a}, \mathrm{b}} \quad$ Claudia Traidl-Hoffmann $^{\mathrm{b}} \quad$ Annette Albert $^{\mathrm{a}} \quad$ Regina Kerzl $^{\mathrm{a}}$ \\ Stephanie Rombold ${ }^{\mathrm{a}}$ Ulf Darsow ${ }^{\mathrm{a}}$ Bernadette Eberlein ${ }^{\mathrm{a}} \quad$ Thilo Jakob $^{c}$ Johannes Ring ${ }^{\mathrm{a}}$ \\ Rüdiger Hein ${ }^{a}$ \\ a Department of Dermatology and Allergy, Technical University, and ${ }^{b}$ ZAUM - Center for Allergy and Environment,

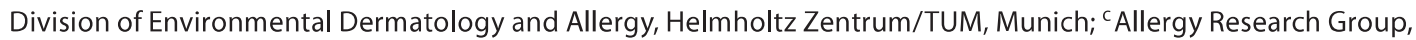 \\ University Medical Center Freiburg, Freiburg, Germany
}

\section{Key Words}

Dermis · Metaplasia, lipomatous .

Cutaneous inflammation

\begin{abstract}
A 69-year-old woman with a history of acute generalized exanthematic pustulosis (AGEP) caused by metamizole is described. Furthermore, she had suffered from an untreated psoriasis since the age of 20 . After an adequate therapy of both psoriasis and AGEP, yellow-brownish, static, coalescing, lucent nodules on the thighs and upper arms became apparent. Histology of skin biopsies revealed a prominent band of mature adipocytes in the dermis. We diagnosed a lipomatous metaplasia of the dermis and hypothesize that this metaplasia occurred as a consequence of the severe and chronic inflammation of the skin.
\end{abstract}

A 69-year-old woman was admitted to our hospital with a suberythrodermia twice within 2 months. She showed a generalized pustular exanthema on the head, trunk and in the intertriginous areas, accompanied by fever $>39^{\circ} \mathrm{C}$, leukocytosis of about $30 \times 10^{3} / \mu$ l and increased erythrocyte sedimentation rate $(70 \mathrm{~mm} / \mathrm{h})$. His- tological examination of skin biopsies taken from the abdomen revealed subcorneal and spongiform pustules which led us to the diagnosis of acute generalized exanthematic pustulosis (AGEP). The medical history revealed that she had received metamizole the day before occurrence of the skin rash in both cases. In addition, concerning the first episode, diclofenac was applied intramuscularly 2 days before pustules became apparent. After treatment of the first episode of the AGEP with corticosteroids (prednisolone intravenously, initial dose $250 \mathrm{mg}$ in declining therapy scheme for 20 days), the cutaneous manifestations declined.

Now static, coalescing lucent nodules ranging from red to yellow-brownish appeared (fig. 1). They were located preferentially on the thighs and upper arms. According to the patient's assertion she had never noticed these skin alterations until a few days before. Notably, the skin rash was neither itchy nor painful. Histology of skin site biopsies of both thighs and upper arms showed an atrophic epidermis with a washy basal membrane zone, a sparse lymphocytic perivascular infiltrate around the upper plexus and a prominent vascularization of the papillary dermis. Right beneath the papillary dermis, a well-vascularized band of fatty tissue consisting of mature adipocytes was detected (fig. 2) Immunohistochemical stainings of CD68 excluded postinflammatory xanthomization, while S100 staining marked mature adipocytes (fig. 3). We diagnosed a lipomatous metaplasia, possibly as a consequence of the severe and chronic inflammation of the skin due to psoriasis and AGEP.

The further medical history revealed that she had suffered from psoriasis since the age of 20. The chronically relapsing psoriasis was tolerated by the patient. An appropriate antipsoriatic therapy was rejected except for a tar-containing topical drug in the 1950s. The medical history did not reveal any allergy, especially not one against drugs.

Besides adiposity, the physical examination was without pathological findings. Laboratory tests of peripheral blood showed normal parameters of the liver and kidneys; serum lipids were in the normal range with subnormal triglycerides. Immunoelectrophoresis and phenotyping of peripheral lymphocytes were performed without pathological findings. Both an abdominal ultrasound and a computed tomography of the cervix, thorax, abdomen and pelvis revealed no pathological findings. Medical investigations by our cardiology, ear, nose and throat and urology 

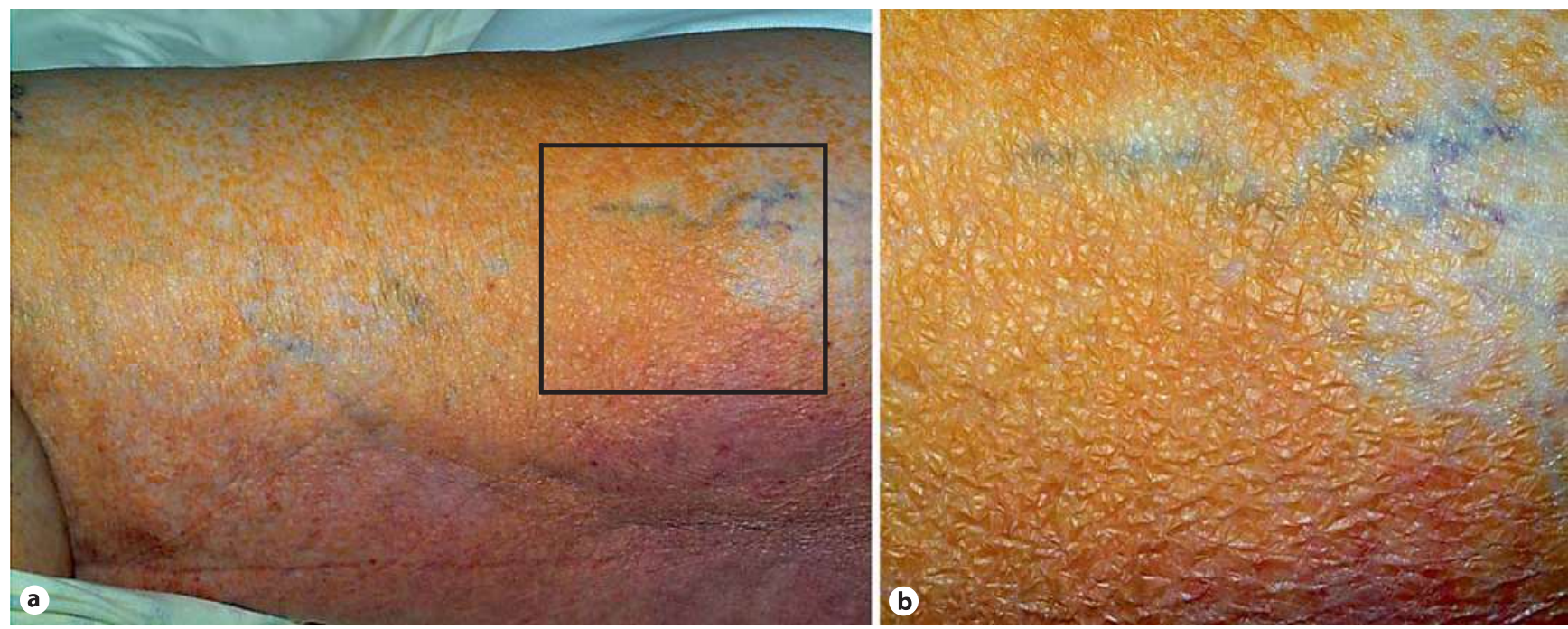

Fig. 1. Clinical aspect of lipomatous metaplasis of the dermis. a Left thigh. $\mathbf{b}$ The inset is shown at higher magnification.
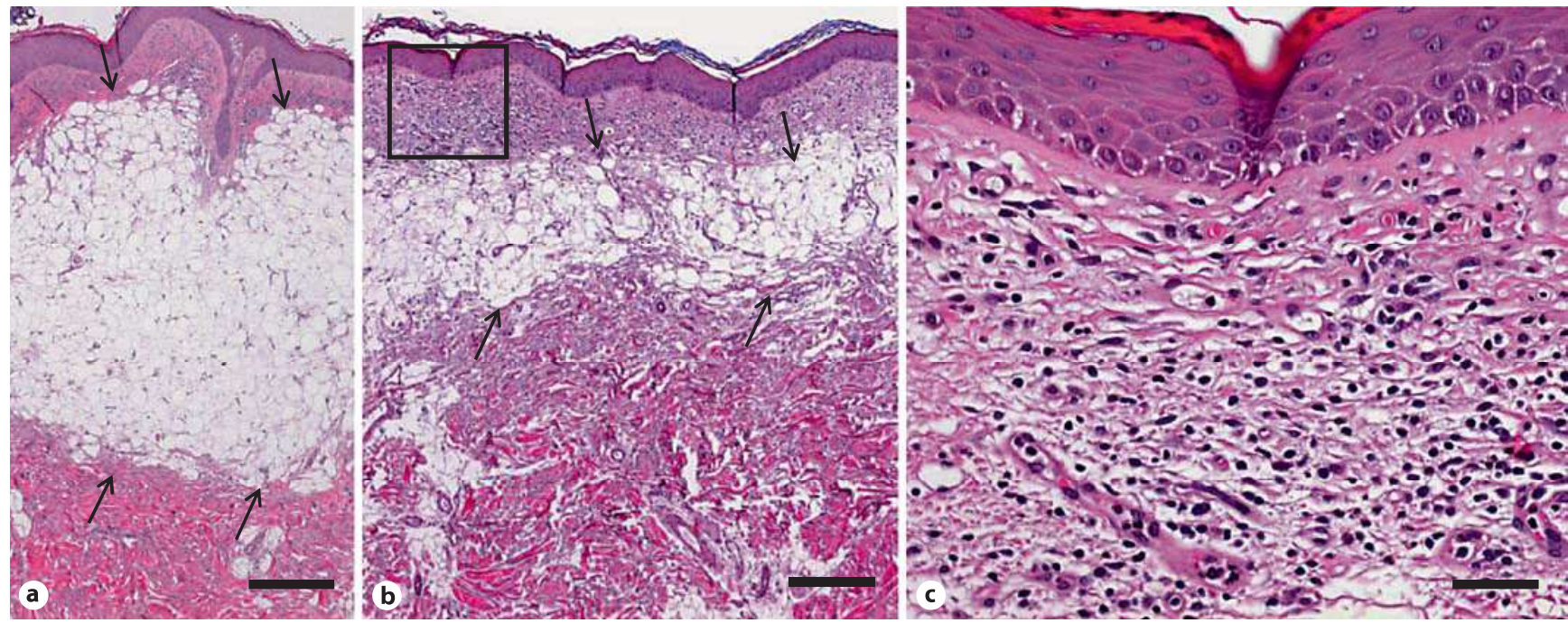

Fig. 2. Histology of lipomatous metaplasia of the dermis (biopsies taken from the left thigh). a An atrophic epidermis, scattered leukocytic infiltrate in the papillary dermis and beneath a prominent band of mature adipocytes. Scale bar $=50 \mu \mathrm{m}$. b Biopsy taken 3 months after the biopsy shown in a. Scale bar $=50 \mu \mathrm{m}$. $\mathbf{c}$ The inset is shown at higher magnification. Scale bar $=25 \mu \mathrm{m}$.

specialists revealed merely hypertension, vertigo of unknown origin and cystic kidneys.

Four weeks after the last ingestion of corticosteroids, the patient underwent allergological diagnosis. Patch testing of analgetics revealed positive reactions to metamizole and indomethacin 48 and $72 \mathrm{~h}$ af- ter application. Prick testing of analgetics was negative and did not show any evidence of a type I allergy. The patient rejected an oral provocation with metamizole and diclofenac. However, acetylsalicylic acid, celecoxib and tramadol were tolerated orally as alternative analgetic drugs.

\section{Discussion}

Metaplasia is a process that occurs commonly after severe and chronic inflammation in the gastrointestinal tract, resulting for example in Barrett's esophagus or gastric ulcers [1]. Lipomatous metaplasia is regularly seen in scars of the left 

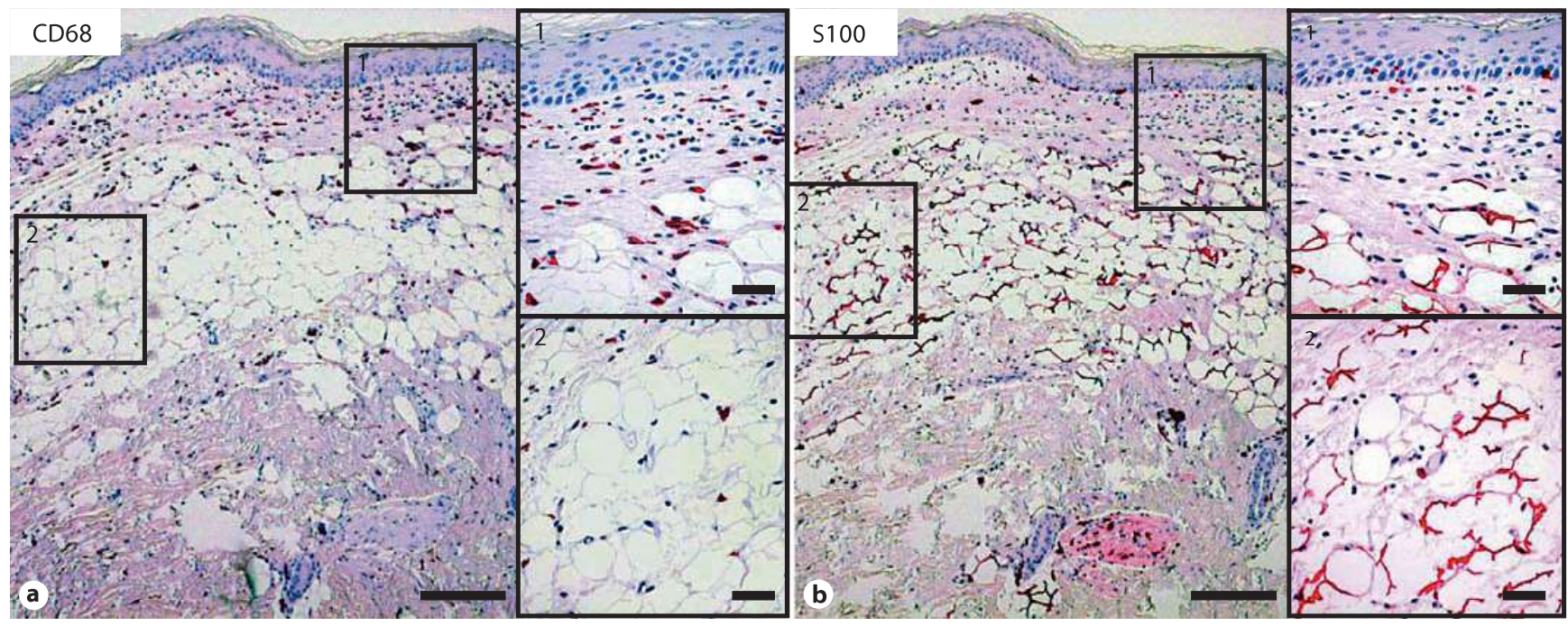

Fig. 3. Immunohistochemical stainings of CD68 (a) and S100 (b) indicate authentic metaplasia. a Only a few macrophages are scattered throughout the dermis. Scale bar $=50 \mu \mathrm{m}$. The insets are shown at higher magnifications. Scale bars $=25 \mu \mathrm{m}$. b Staining of S100 marks mature adipocytes. Scale bar $=50 \mu \mathrm{m}$. The insets are shown at higher magnifications. Scale bars $=25 \mu \mathrm{m}$.

ventricle after an ischemic heart disease [2] as well as in endogenous [3] and exogenous [4] glands and a rare subpopulation of meningiomas [5], but it is not generally associated with chronic inflammation. In the skin, lipomatous metaplasia is very uncommon. It is discussed to play a role in the pathology of the rare lipomatous neurofibromas [6], but a diffuse lipomatous metaplasia of the dermis as seen in our patient has, at least to our knowledge, not been described so far.

The patient suffered from a chronically inflamed skin due to an untreated psoriasis for nearly 50 years. At least twice she developed an AGEP meeting both the clinical [7] and the histological [8] criteria. Following medical examination and positive patch testing of metamizole that has been reported to be a reliable test method
[9], metamizole seems to be the most likely drug causative for this severe immune reaction, although it is not among the most frequent causative drugs responsible for an AGEP [10]. However, besides the most common causative drugs like antibiotics [11] or diltiazem [12], a huge variety of drugs including even corticosteroids [13] have been reported to induce AGEP.

In parallel to the described episodes of severe generalized cutaneous inflammation, a diffuse lipomatous dermal metaplasia was observed for the first time. Authentic metaplasia could be confirmed using immunohistochemical stainings of CD68 and S100: mature adipocytes were clearly positive for S100 [14] whereas CD68-positive cells were scarce within the lipomatous tissue ruling out a postinflammatory xanthomization (fig. 3). This metaplasia is static (fig. 2) and does not cause any symptoms such as pain or itch so far. Whether it could be a risk factor for the development of a tumor, as lipomatous metaplasia has been reported to be a critical step in tumorigenesis [15], remains elusive. We have followed up our patient for 1 year now, taking biopsies every 3 months, and we have not seen any clinical or histological changes so far. With an adequate therapy of the psoriasis and avoidance of the suspected analgetics metamizole and diclofenac, the clinical condition of the skin is stable, as is the dispersal and quantity of the lipomatous metaplasia. We therefore conclude that the chronic and severe inflammation of the skin may have provoked this lipomatous metaplasia of the dermis.

\section{References}

1 Malfertheiner P, Peitz U: The interplay between Helicobacter pylori, gastro-oesophageal reflux disease, and intestinal metaplasia. Gut 2005;54(suppl 1):13-20.

2 Nijveldt R, Marcu CB, van Rossum AC: Lipomatous metaplasia in myocardial infarction detected by cardiovascular magnetic resonance. Heart 2006;92:1337.
3 Finch C, Davis R, Truong LD: Extensive lipomatous metaplasia in bilateral macronodular adrenocortical hyperplasia. Arch Pathol Lab Med 1999;123:167-169.
4 Skalova A, Starek I, Simpson RH, Kucerova V, Dvorackova J, Curik R, Duskova M: Spindle cell myoepithelial tumors of the parotid gland with extensive lipomatous metaplasia: a report of four cases with immunohistochemical and ultrastructural findings. Virchows Arch 2001;439:762-767. 
5 Roncaroli F, Scheithauer BW, Laeng RH, Cenacchi G, Abell-Aleff P, Moschopulos M: Lipomatous meningioma: a clinicopathologic study of 18 cases with special reference to the issue of metaplasia. Am J Surg Pathol 2001;25:769-775.

6 Val-Bernal JF, González-Vela MC: Cutaneous lipomatous neurofibroma: characterization and frequency. J Cutan Pathol 2005;32: 274-279.

7 Prange B, Marini A, Kalke A, Hodzic Avdagic N, Ruzicka T, Hengge UR: Acute localized exanthematic pustulosis (ALEP). J Dtsch Dermatol Ges 2005;3:210-212.

8 Greither A, Hofmann N, Kurz E: Acute generalized pustulosis: a clinico-histopathological study on differential diagnosis. Z Hautkr 1981;56:1209-1216.
9 Ozkaya-Bayazit E: Topical provocation in fixed drug eruption due to metamizole and naproxen. Clin Exp Dermatol 2004;29:419422.

10 Saissi EH, Beau-Salinas F, Jonville-Béra AP, Lorette G, Autret-Leca E, Centres Régionaux de Pharmacovigilance: Drugs associated with acute generalized exanthematic pustulosis. Ann Dermatol Vénéréol 2003;130:612618.

11 Haeusermann P, Scherer K, Weber M, Bircher AJ: Ciprofloxacin-induced acute generalized exanthematous pustulosis mimicking bullous drug eruption confirmed by a positive patch test. Dermatology 2005;211:277280.

12 Gesierich A, Rose C, Broecker EB, Trautmann A, Leverkus M: Acute generalised exanthematous pustulosis with subepidermal blisters of the distal extremities induced by diltiazem. Dermatology 2006;213:48-49.
13 Buettiker U, Keller M, Pichler WJ, Braathen LR, Yawalkar N: Oral prednisolone induced acute generalized exanthematic pustulosis due to corticosteroids of group A confirmed by epicutaneous testing and lymphocyte transformation tests. Dermatology 2006; 213:40-43.

14 Atanassova P: Immunohistochemical expression of S-100 protein in human embryonal fat cells. Cell Tissues Organs 2001;169: 355-360.

15 Izumi M, Serizawa H, Iwaya K, Takeda K, Sasano H, Mukai K: A case of myxoid adrenocortical carcinoma with extensive lipomatous metaplasia. Arch Pathol Lab Med 2003; 127:227-230. 